\title{
THE VARIATIONS IN LIGHT CURVES OF CONTACT BINARY AU SER
}

\author{
LI ZONG-YUN DING YUE-RONG \\ Department of Astronomy, Nanjing University, Nanjing 210093, China \\ email: zyli@netra.nju.edu.cn
}

Photometry of AU Ser, a contact binary in poor thermal contact, was made using the 1-meter reflector of the Yunnan Observatory, China, in 1991,1992 and 1995. Our observations, together with Binnendijk's(1972), show variations in light curves similar to ones of VW Cep:

1) Changes in sign and value of $O^{\prime}$ Connell effect. According to Binnendijk(1972), the maximum following primary minimum was higher than the other maximum, i.e., the O'Connell effect was positive. In our 1991 and 1992 light curves it became negative. After three years, however, the situation has reversed again.

2) Variations of the difference between minimum depths. The difference in 1991 was smaller than that in 1969 and 1970. It got smallest in 1992 and largest in 1995. The difference increases by about $0.3 \mathrm{mag}$ in three years, which is hard to explained.

3) The phase shift. The observed minimum phase in 1991, 1992 and 1995 have progressively displaced forward with respect to computed minimum phase according to Binnendijk's ephemeris. The O-C diagram seems to indicate a sudden decrease of orbital period after 1970. Also, short period oscillations have been found at frequencies of $0.0003,0.005$ and $0.0075 \mathrm{~Hz}$. Flickering. with an average amplitude of about $0.05 \mathrm{mag}$, can be seen in the light curves. The power spectral analysis suggest the existence of oscillations. It is unusual that such oscillations exist in a contact binary. 